\title{
Journal of Clinical Immunology \\ A report of novel STIM1 deficiency and 6 year follow up of two previous cases associated with mild immunological phenotype \\ --Manuscript Draft--
}

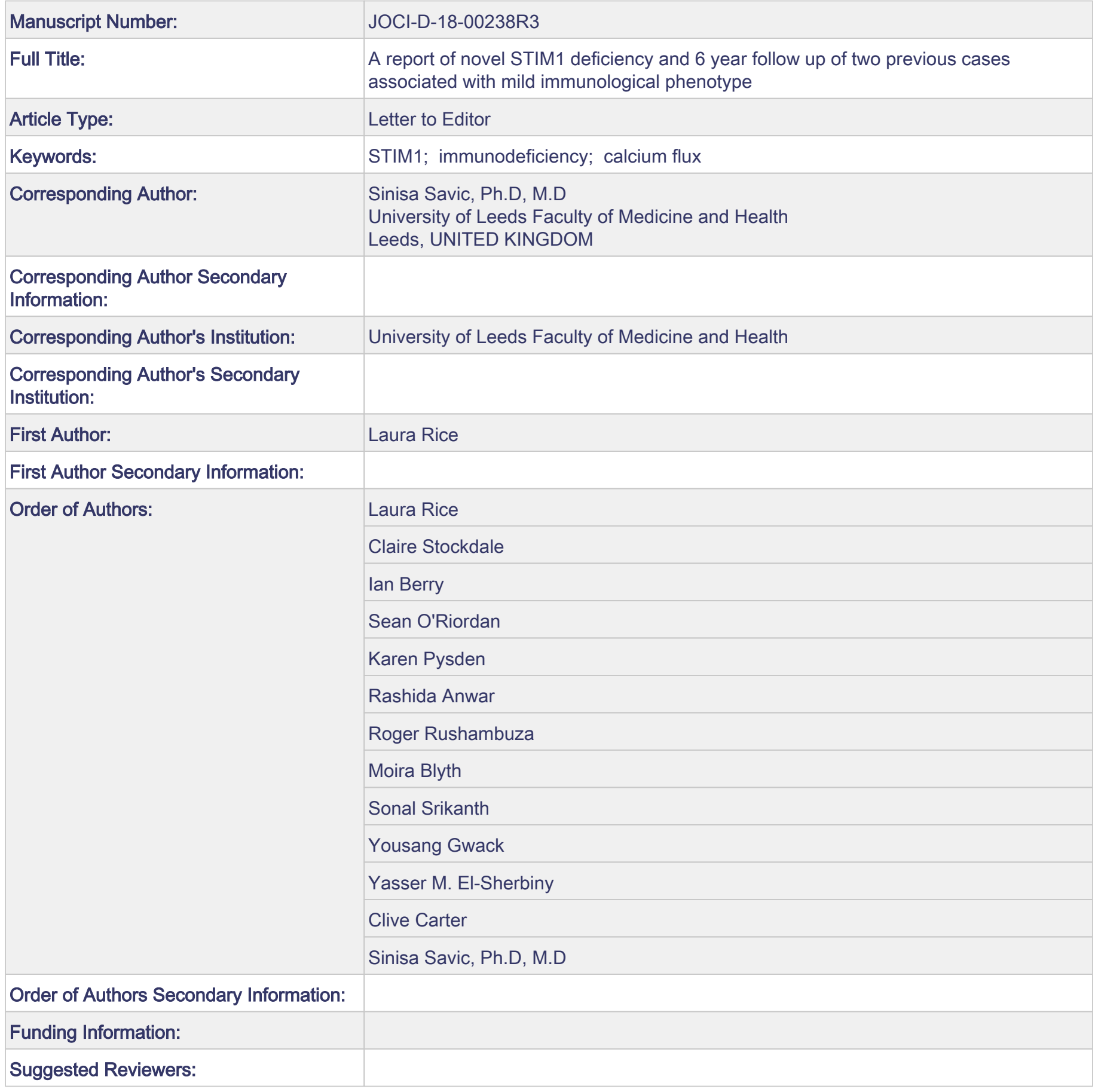




\section{A report of novel STIM1 deficiency and 6 year follow up of two previous cases associated with mild immunological phenotype}

Laura Rice ${ }^{1^{*}}$, Claire Stockdale ${ }^{2^{*}}$, lan Berry ${ }^{3}$, Sean O'Riordan ${ }^{4}$, Karen Pysden ${ }^{5}$, Rashida Anwar $^{1}$, Roger Rushambuza 6 , Moira Blyth ${ }^{3}$, Sonal Srikanth ${ }^{7}$, Yousang Gwack, Yasser M. El-Sherbiny ${ }^{8,9,10}$ Clive Carter $^{2}$, Sinisa Savic ${ }^{2,10}$

${ }^{1}$ Leeds Institute of Medical Research at St James's, University of Leeds, Wellcome Trust Brenner Building, St James's University Hospital, Beckett Street, Leeds, UK

${ }^{2}$ Department of Clinical Immunology and Allergy, St James's University Hospital, Beckett Street, Leeds, UK

${ }^{3}$ Yorkshire Regional Genetics Service, Chapel Alleron Hospital, Leeds, UK

${ }^{4}$ Department of Paediatric Immunology, Leeds General Infirmary, Leeds, UK

${ }^{5}$ Department of Paediatric Neurology, Leeds General Infirmary, Leeds, UK

${ }^{6}$ Department of Radiology, Calderdale and Huddersfield NHS Foundation Trust, UK

7 Department of Physiology, David Geffen School of Medicine, UCLA, Los Angeles, CA 90095, USA

${ }^{8}$ Department of Biosciences, School of Science and Technology, Nottingham Trent University,

Nottingham, UK.

${ }^{9}$ Clinical Pathology Department, Faculty of Medicine, Mansoura University, Egypt.

${ }^{10}$ National Institute for Health Research-Leeds Biomedical Research Centre and Leeds Institute of Rheumatic and Musculoskeletal Medicine (LIRMM), Wellcome Trust Brenner Building, St James's University Hospital, Beckett Street, Leeds, UK

*Equal contribution

Correspondence to: Dr Sinisa Savic, s.savic@leeds.ac.uk 
To the Editor,

Loss of function or null mutations of Stromal interaction molecule 1 (STIM1) are known to cause early-onset combined immunodeficiency (CID) disease with recurrent and chronic infections, autoimmunity, haemolytic anaemia, ectodermal dysplasia, muscular weakness and myalgia (1). STIM1 and ORAl1 form the calcium release-activated calcium (CRAC) channels and are involved in calcium signalling, which is especially important in $\mathrm{T}$ cells for activation, proliferation and cytokine production (2). ORAl1 forms the pore in the plasma membrane and STIM1 is a calcium sensor protein that activates the ORAl1 when the endoplasmic reticulum (ER) $\mathrm{Ca}^{2+}$ stores are depleted.

STIM1-deficient patients have impaired $T$ cells and NK cell function, but usually a normal distribution of the major immune cell types, including T cells, B cells and natural killer (NK) cells with the T cell repertoire that is normally comparable to healthy individuals (3). STIM1 deficiency results in no store-operated calcium entry (SOCE) in T cells and as a result the patient's cells cannot respond appropriately to $\mathrm{T}$ cell receptor (TCR) activation or pharmacological agents such as ionomycin, thapsigargin (TG), which typically trigger $\mathrm{Ca}^{2+}$ influx (1).

Recently a new biological role for STIM1 has been identified. STIM1 was found to act as a negative regulator for STimulator of INterferon Genes (STING). STIM1 was shown to inhibit STING trafficking by physically interacting with STING and retaining it at the ER membrane. This interaction is important in maintaining STING in an inactive state (4). Several STING mutations which have previously shown to cause an autoinflammatory condition named STING-associated vasculopathy with onset in infancy (SAVI), appear to exert their dominant effects by weakening the interaction between STIM1 and STING (4).

Here we describe a 5-year-old boy of consanguineous Pakistani background with overlapping clinical features of CID and autoinflammatory disorder. The boy was initially referred to pediatric immunology services with a history of recurrent sinopulmonary infections. The patient was born at full term by normal vaginal delivery following a normal pregnancy. An inguinal hernia was noted when six days old and was repaired the same day. He initially fed well. At six months of age it was noted that he was quieter and delayed in his development. He sat at 18 months and walked at 3 years and crawls upstairs using his head as support. Currently, at 5 years he is alert and engaging but has speech delay putting 2-3 words together. $\mathrm{He}$ 
can scribble and draw circles. He was fully toilet-trained at 3 years. He is in mainstream education but requires additional support.

When examined at 5 years, he weighed $11.5 \mathrm{~kg}(-5 S D)$, with a height of $94.2 \mathrm{~cm}(-4 S D)$ and head circumference of $46.1 \mathrm{~cm}(-4 S D)$. He is slim, with reduced proximal upper limb muscle bulk and globally in his lower limbs, lordosis and is hypermobile across all his joints. He has proximal weakness leading to a waddling gait, and uses a Gowers manoeuvre to stand. He has no facial weakness, no opthalmoparesis but has fixed dilated pupils.

The patient infection history included recurrent sinopulmonary infections with one episode of pneumonia requiring hospital admission and intravenous antibiotics. $\mathrm{He}$ had received all his primary vaccinations, including MMR, without significant complications. He was hospitalized for a complicated primary varicella zoster infection due to bacterial suprainfection requiring antibiotic treatment. In addition, he had several hospitalizations with suspected infections, but despite having documented fevers, no apparent infective cause was found and on several occasions, he recovered without receiving antibiotics. A full dental clearance was performed due to recurrent tooth infections and tooth enamel deficiency. Other characteristics include, ichthyosis, anhidrosis and low zinc levels $[7.8 \mu \mathrm{mol} / /$ (reference range 10.3-18.1)]. Although he was described to have severe eczema his skin eruptions mainly affected the palms, soles of the feet and cheeks, (cold exposed areas), and would start with blistering sterile pustular psoriasiform rash, eventually resulting in skin desquamation. Zinc replacement did not lead to improvement, and there was minimal benefit from application of topical steroid. The patient also had pronounced nail dystrophy (Figure 1). There was no history of Raynaud's phenomena.

He has two other siblings who are fit and healthy. The pedigree is shown in Figure $2 \mathrm{~A}$.

The initial investigations including full blood count, creatinine kinase, screen for inherited metabolic diseases and MRI scan of the head were all normal. The immunological work up showed essentially normal numbers of T, B and NK cells. More detailed phenotyping revealed, detectable regulatory $\mathrm{T}$ cells and unremarkable memory B cell profile. Immunoglobulin profile (IgG, IgA, IgM and IgG subclasses) was normal with marginally elevated total $\lg \mathrm{E}$. The levels of specific antibodies to 
tetanus and Haemophilus influenzae type $\mathrm{b}$ (Hib) were adequate and showing a antibody levels were reduced, however, following a challenge vaccination with pneumovax, the levels increased well above the protective titre (from 6.2 to 120.7 $\mu \mathrm{g} / \mathrm{ml})$

Considering some of the clinical features, diagnosis of STIM1 or ORAl1 deficiency was suspected. The parents consented for genetic testing. A modified exome sequencing approach was performed using Agilent SureSelectXT with All Exon v5 capture library and sequenced on Illumina HiSeq 3000 for 2x150-bp pairedend sequencing. Genetic testing identified a homozygous deletion in STIM1 NM_003156.3:c.478del, p.(Ser160ValfsTer15), and a homozygous CANT1 mutation NM_001159772.1:c.676G>A, p.(Val226Met) (Figure 2A). This known pathogenic CANT1 mutation has previously been described in multiple patients with "Kimvariant" Desbuquois dysplasia, usually in compound heterozygosity with another mutation $(5,6)$. These patients have normal clinical examination but advanced carpal age, elongated phalanges and short metacarpals on radiological examination. Only one patient, reported to have "Kim-variant" Desbuquois dysplasia, was homozygous for the CANT1 c.676G>A mutation but little phenotypic information was provided $(5,6)$. The same homozygous mutation has also been described in a single patient with autosomal recessive multiple epiphyseal dysplasia (7). That patient did not have many of the features of "Kim-variant" Desbuquois dysplasia but had developed the degenerative arthrosis of the hands and spine by the age of 25 years.

The novel single nucleotide deletion at c.478 in STIM1 results in a frameshift such that translation terminates prematurely within the sterile alpha motif (SAM), the region regulating stability within STIM proteins. The truncated polypeptide of 173 amino acids (wild type STIM1 polypeptide has several splice variants, one of which is 791 amino acids, another form is 685) lacks important functional domains of STIM1, including the transmembrane region and the CRAC activation domain (CAD). We used western blot to investigate the expression of STIM1 in the patient's (P1) PBMCs, and failed to detect either the full length or the truncated variant of STIM1. However, we did detect expression of STIM2, a homologue of STIM1. The former was not expressed in PBMCs from HC (Figure 1B). 
The functional impact of these mutations was investigated further. Regarding the STIM1 variant we performed flow cytometry $T$ receptor spectraphenotyping and showed essentially normal TCR repertoire (Figure 1 Suppl). A single $\mathrm{Vb}$ region (Vb7.2) was missing from patient's T cells, however the clinical significance of this is unknown. Proliferative responses to Phytohaemagglutinin (PHA) were adequate (although reduced at high concentrations) and the response to anti-CD3 was preserved (Figure 2A Suppl). There was significantly reduced upregulation of CD25 following anti-CD3 in-vitro stimulation whilst upregulation of HLA-DR and CD69 was normal (Figure2B suppl). Measurement of store-operated $\mathrm{Ca}^{2+}$ entry in patient $\mathrm{T}$ cells revealed a complete lack of calcium influx in response to anti-CD3/anti-CD28 stimulation and TG, as seen in Figure 2C.

A skeletal survey was performed to investigate the impact of the CANT1 variant on the patient's phenotype. His overall small stature was consistent with previous reports regarding the effects of this CANT1 variant. His skeletal survey revealed the abnormal early maturation of carpal bones seen in CANT1-related disorders. It also showed a gentle curve scoliosis of the cervicothoracic spine, which is in keeping with CANT1-related disorders. The skeletal survey did not convincingly demonstrate the so-called monkey-wrench deformity of the femoral necks, shortened metacarpal bones, and other abnormalities which have previously been described in patients with Desbuquois dysplasia $(8,9)$ (Figure 3). Some of the radiological features are progressive and may appear at a later date but the skeletal phenotype is more in keeping with the patient described as having MED (7). That patient was also homozygous for the CANT1 c.676G>A and it has already been proven that this mutation significantly reduces, but does not eliminate, the nucleotidase activity of CANT1(5). In comparison, most patients described as having "Kim-variant" Desbuquois dysplasia have this mutation alongside a second mutation anticipated to cause a premature stop codon or shown to cause more significantly reduced CANT1 nucleotidase activity (5). As has already been hypothesised, it is likely that homozygosity for the p.(Val226Met) mutation in CANT1 causes a less severe phenotype than the "Kim-variant" Desbuquois dysplasia (7), which occurs when a more damaging mutation is present on the other allele.

We have previously reported two other cases with a homozygous mutation in STIM1, resulting in impaired store-operated calcium entry, reduced natural killer and 
T cell function, but without overt clinical immunodeficiency (10) (Table 1). We arranged a follow up visit to assess their clinical progress and repeat immunological assessment following their initial investigations in 2012. From the immunological perspective, 6 years later, both cousins were generally well and had not suffered from any serious, frequent or opportunistic infections. They both continued to experience some problems with anhydrosis and mild muscle weakness but were otherwise unaffected by their condition. They did not report any new symptoms. Repeat immunological assessment showed that $\mathrm{V}: 3$ (older cousin as designated in the original publication) had persistent CD8 lymphopenia ( $8 \%, 86 \mathrm{cells} / \mu \mathrm{l})$, new mild B cell lymphopenia (88 cells $/ \mu$ l) (ref: 100-500 cells $/ \mu$ l), but also normal immunoglobulin profile with adequate levels of specific antibodies to Hib, tetanus and pneumococcus. Furthermore, repeated PHA and anti-CD3 $\mathrm{T}$ cell stimulation indicated normal responses. TCR repertoire, assessed by $T$ receptor spectraphenotyping, showed no abnormalities. The results of the tests on the second cousin (V:2) are shown in Table 1. These are essentially unchanged apart from the anti-nuclear antibody test which on this occasion was negative.

We have previously shown that monocytes and peripheral blood mononuclear cells (PBMCs) from the patient (P1) with the novel STIM1 deletion mutation have significantly increased interferon-stimulated gene (ISG) expression compared to healthy controls $(\mathrm{HC})(4)$. This was consistent with expected loss of STIM1-mediated STING inhibition (4). We wanted to check if this STIM1 function is also affected in patients harboring p.L74P STIM1 variant. We obtained blood from the patient (V:2) and PBMCs were separated by gradient separation using Lymphoprep (Stem Cell Technologies) and monocytes were purified from PBMCs using a monocyte separation kit II (\# 130-091-153, Miltenyi Biotec). The ISG expression was measured using TaqMan probes (for details please see supplements). While ISG expression in PBMCs and monocytes from V:2 was reduced compared to what we previously have shown for P1(4) there was significant increase in several ISG compared to HC, suggesting that the p.L74P variant has effect on STIM1-mediated STING inhibition (Figure 4). We have previously shown that overexpression of p.L74P variant in HEK293 cells results in preferential localization of the mutated protein to puncta rather than the ER (10). Using Western blot and two sets of anti-STIM 1 antibodies (one specific for the $\mathrm{N}$-terminal and the other for protein $\mathrm{C}$-terminal, for details please 
see supplements) we failed to detect any STIM1 expression in V:2 PBMCs. This suggest that p.L74P mutation under physiological conditions affects the protein stability, resulting in reduced expression, and therefore reduced inhibition of STING.

The new patient we describe in this report has two monogenic conditions, STIM1 deficiency and CANT1-related disorder. However, most of the clinical manifestations are due to STIM1 deficiency. The CANT1 variant will be responsible for the short statue, mild scoliosis and advanced carpal age but other features such as premature arthritis may develop with time. On the other hand, the nature of genetic defect found in STIM1 is more severe (deletion compared to amino acid substitution in CANT1) and the non-immunological features typically associated with STIM1 deficiency (anhydrosis, amelogenesis imperfecta and muscle weakness) are fully expressed. Furthermore, biochemical studies of the new STIM1 variant, clearly showed loss of expression of full length STIM1 (4).

STIM 1 deficiency is still quite rare, however with increasing number of cases being reported world-wide a more complete picture of the clinical phenotypes associated with this disorder is beginning to emerge. The homozygous mutations resulting in amino acid substitution and total lack of protein expression have both been described. Although there are too few cases to determine if there is genotypephenotype correlation in this condition (Table 2), the only fatalities associated with STIM1 deficiency have only been reported with the mutations resulting in total loss of the protein expression (LOE) $(1,11,12)$, however, SCID-like phenotypes have been described in patients where STIM1 mutations lead to loss of function without LOE $(12,15)$.

The non-immunological features of the case we report here overall fit with the classical STIM1 deficiency due to enamel deficiency, anhydrosis and muscle weakness; but the mild immunological phenotype, in a patient lacking full length STIM1 expression is a novel presentation. Furthermore, this patient has several clinical features suggestive of autoinflammatory complications. Although he does not have pulmonary involvement (fibrosis) or severe ulceration and necrosis of the skin, which are typical for SAVI, he did have pustular rash developing at the cold exposed areas of the skin and acral surfaces, which have been reported in this condition (16). $\mathrm{He}$ also had a history of unexplained fevers, however, to an extent, this could be explained by anhydrosis. Interestingly, patient V:2, who also has increased ISG, does not have any clinical features to suggest autoinflammatory condition. 
Therefore, the effect of SAVI disinhibition in STIM1 deficiency on clinical phenotype is complex and difficult to predict. Nevertheless, overlap between PID and systemic autoinflammatory disorders is increasingly being recognized, and this case adds to this growing disease area.

There are scant long-term outcome data for patients with STIM1 deficiency. The patients we reported first in 2012 and reinvestigated now in 2018 have not seemingly developed any overt immunological problems and reassuringly the repeat investigations of their immune system do not show any significant deterioration. It is interesting that lack of calcium flux seen in the patients' $T$ cells, either due to total lack of full length STIM 1 expression or due to truncated STIM1, has not resulted in a more profound immunodeficiency. We assume that a form of compensatory mechanism must be in place to account for this outcome. One possibility is that other related proteins such as STIM2, a homologue of STIM1, might provide this role. Indeed, when we examined expression of STIM2 in PBMCs from P1 and V:2, the expression of the protein was increased compared to HC, in fact HC PBMCs did not show any expression of STIM2 under resting conditions. In addition, high interferon drive was detected in both STIM1 deficient cases, and this might offer some protection against viral pathogens. Lastly, considering immunological and nonimmunological features of STIM1 deficiency, this condition should be thought of as another form of anhidrotic ectodermal dysplasia with immunodeficiency (EDA-ID), similarly to what has recently been proposed for ORA1 deficiency (17).

Disclosure of potential conflict of interest: The authors declare that they have no relevant conflicts of interest.

Acknowledgements

This research is supported by grant from CSL Behring and by the National Institute for Health Research (NIHR) Leeds Biomedical Research Centre. The views expressed are those of the author(s) and not necessarily those of the NHS, the NIHR or the Department of Health. 
Reference

1. Picard C, McCarl C-A, Papolos A, et al. STIM1 Mutation Associated with a Syndrome of Immunodeficiency and Autoimmunity. The New England journal of medicine. 2009;360(19):1971-1980. doi:10.1056/NEJMoa0900082.

2. Feske S, Prakriya M, Rao A, Lewis RS. A severe defect in CRAC $\mathrm{Ca}^{2+}$ channel activation and altered $\mathrm{K}^{+}$channel gating in $\mathrm{T}$ cells from immunodeficient patients. The Journal of Experimental Medicine. 2005;202(5):651-662. doi:10.1084/jem.20050687.

3. Feske S, Picard C, Fischer A. Immunodeficiency due to mutations in ORAl1and STIM1. Clinical immunology (Orlando, Fla). 2010;135(2):169-182. doi:10.1016/j.clim.2010.01.011.

4. Srikanth S, Woo JS, Wu B, El-Sherbiny YM, Leung J, Chupradit K, Rice L, Seo GJ, Calmettes G, Ramakrishna C, Cantin E, An DS, Sun R, Wu T-T, Jung JU, Savic S, Gwack Y. STIM1 regulates type I interferon response by retaining STING at the endoplasmic reticulum. Nat Immun 2018- accepted for publication

5. Furuichi T, Dai J, Cho T, et al, CANT1 mutation is also responsible for Desbuquois dysplasia, type 2 and Kim variant, Journal of Medical Genetics 2011;48:32-37. doi:10.1136/jmg.2010.080226

6. Kim OH, Nishimura G, Song HR, Matsui Y, Sakazume S, Yamada M, Narumi Y, Alanay $Y$, Unger S, Cho TJ, Park SS, Ikegawa S, Meinecke P, Superti-Furga A. A variant of Desbuquois dysplasia characterized by advanced carpal bone age, short metacarpals, and elongated phalanges: report of seven cases. Am J Med Genet A. 2010;152A(4):875-85

7. Balasubramanian K, Li B, Krakow D, Nevarez L, Ho PJ, Ainsworth JA, Nickerson DA, Bamshad MJ, Immken L, Lachman RS, Cohn DH. MED resulting from recessively inherited mutations in the gene encoding calcium-activated nucleotidase CANT1. Am J Med Genet A. 2017;173(9):2415-2421. doi:10.1002/ajmg.a.38349

8. Nizon M, Huber C, De Leonardis F, Merrina R, Forlino A, et al Further Delineation of CANT1 Phenotypic Spectrum and Demonstration of Its Role in Proteoglycan Synthesis. Hum Mutat 2012; 33(8): 1261-1266.

9. Bui C, Huber C, Tuysuz B, Alanay Y, 1 Mutations in Desbuquois Dysplasia Type 2. Am J Hum Genet. 2014 Mar 6; 94(3): 405-414.

10. Parry DA, Holmes TD, Gamper N, et al. A homozygous STIM1 mutation impairs storeoperated calcium entry and natural killer cell effector function without clinical immunodeficiency. The Journal of Allergy and Clinical Immunology. 2016;137(3):955-957.e8. doi:10.1016/j.jaci.2015.08.051.

11. Byun M, Abhyankar A, Lelarge $\mathrm{V}$, et al. Whole-exome sequencing-based discovery of STIM1 deficiency in a child with fatal classic Kaposi sarcoma. The Journal of Experimental Medicine. 2010;207(11):2307-2312. doi:10.1084/jem.20101597.

12. Fuchs S, Rensing-Ehl A, Speckmann C, et al. Antiviral and regulatory T cell immunity in a patient with STIM1 deficiency. Journal of Immunology (Baltimore, Md : 1950). 2012;188(3):1523-1533. doi:10.4049/jimmunol.1102507.

13. Schaballie H, Rodriguez R, Martin E, Moens L, Frans G, Lenoir C, Dutré J, Canioni D, Bossuyt X, Fischer A, Latour S, Meyts I, Picard C. A novel hypomorphic mutation in STIM1 results in a late-onset immunodeficiency. J Allergy Clin Immunol. 2015;136(3):816-819.e4. doi: 10.1016/j.jaci.2015.03.009 
14. Wang S, Choi M, Richardson AS, Reid BM, Seymen F, Yildirim M, et al. STIM1 and SLC24A4 are critical for enamel maturation. J Dent Res 2014;12:94S-100S.

15. Maus M, Jairaman A, Stathopulos PB, Muik M, Fahrner M, Weidinger C, Benson M, Fuchs S, Ehl S, Romanin C, Ikura M, Prakriya M, Feske S. Proc Natl Acad Sci U S A. Missense mutation in immunodeficient patients shows the multifunctional roles of coiled-coil domain 3 (CC3) in STIM1 activation. 2015;112(19):6206-11

16. Canna SW, Goldbach-Mansky R. New monogenic autoinflammatory diseases--a clinical overview Semin Immunopathol. 2015;37(4):387-94

17. Lian J, Cuk M, Kahlfuss S, Kozhaya L, Vaeth M, Rieux-Laucat F, Picard C, Benson MJ, Jakovcevic A, Bilic K, Martinac I, Stathopulos P, Kacskovics I, Vraetz T, Speckmann C, EhI S, Issekutz $\mathrm{T}$, Unutmaz D, Feske S. ORAl1 mutations abolishing store-operated $\mathrm{Ca}^{2+}$ entry cause anhidrotic ectodermal dysplasia with immunodeficiency. J Allergy Clin Immunol. 2018;142(4):1297-1310.e1 
Figure 1. Skin desquamation on the palms and nail dystrophy. These pictures were taken following initial blistering of the skin

Figure 2. Patient with STIM1 deficiency. A) Mutation segregation (N/A- not available, the genotypes of two siblings are not available, but both are healthy and neither have any clinical features to suggest STIM1 or CANT1 deficiency). B) Western blot showing expression of STIM1and STIM2 in PBMC from $\mathrm{HC}, \mathrm{P} 1$ and $\mathrm{V}: 2$, representative blot from 2 separate experiments, *Non-specific binding C) Calcium flux in Patient $\mathrm{T}$ cells compared to healthy control $(\mathrm{HC})$. T cells were incubated for 1 hour at $37^{\circ} \mathrm{C}$ in $0 \% \mathrm{CO}_{2}$ with $2 \mu \mathrm{M}$ fura-2 AM in standard bath solution (SBS) with $0.01 \%$ pluronic acid (Invitrogen). Cells were seeded at $5 \times 10^{5}$ cells/well. T cells were stimulated for 90 seconds by either Thapsigargin (TG) at $1 \mu \mathrm{M}$ or the TCR was activated by soluble CD3 at $1 \mu \mathrm{g} / \mathrm{ml}$ (Clone OKT3). For TCR stimulation the plate was coated with anti-CD28 $(1 \mu \mathrm{g} / \mathrm{ml}$, Clone CD28.2). Fura-2 was excited at $340 \mathrm{~nm}$ and 380 $\mathrm{nm}$ and emission was collected at $510 \mathrm{~nm}$. Measurements were taken on a 96-well fluorescence plate reader (FlexStation III, Molecular Devices)

Figure 3. The image on the left (A) is a DP x-ray of the hand of a normal 5 year old boy. The ossification centre of the ulnar epiphysis (ue) is not visible and the scaphoid (S) and trapezium and trapezoid $(\mathrm{T})$ ossifications centres are very small. The study was performed following penetrating trauma and the density between the middle and ring metacarpal bones is a glass foreign body. Compare with the appearances of our patient's hand on the right (B) where the ulnar epiphysis ossification centre is clearly visible and the radial sided carpal bones are nearly fully formed with appearances more typical of an 8 or 9 year old boy. C) Xray of thoracolumbar Spine". It demonstrates a gentle scoliosis of the cervicothoracic spine.

Figure 4. Interferon-stimulated gene (ISG) expression in patients' and healthy controls (HC) monocytes. V:2, patients with homozygous pL74P STIM1 mutation. Statistical analysis was carried out using two-tailed/unpaired Student's t-test ${ }^{*} p<0.05{ }^{* *} p<0.005$ and ${ }^{* * *} p<0.0005$

Figure 1. Suppl. TCR repertoire as assessed by T receptor spectraphenotyping. The arrow is showing a missing Vb7.2 expression on patients' T cells.

Figure 2. Suppl. A) Phytohaemagglutinin (PHA, 3 differed concentrations 5,10 and $100 \mu \mathrm{g} / \mathrm{ml}$ ) and anti-CD3 T cell proliferation (P1 red 2 separate experiments) Healthy controls (HC black) X3 B)_CD69, HLA-DR and CD25 expression on T cells form the patient (P1 red) and $3 \mathrm{HC}$ black following stimulation with anti-CD3 for 48 hours 
Table 1 Immunological findings

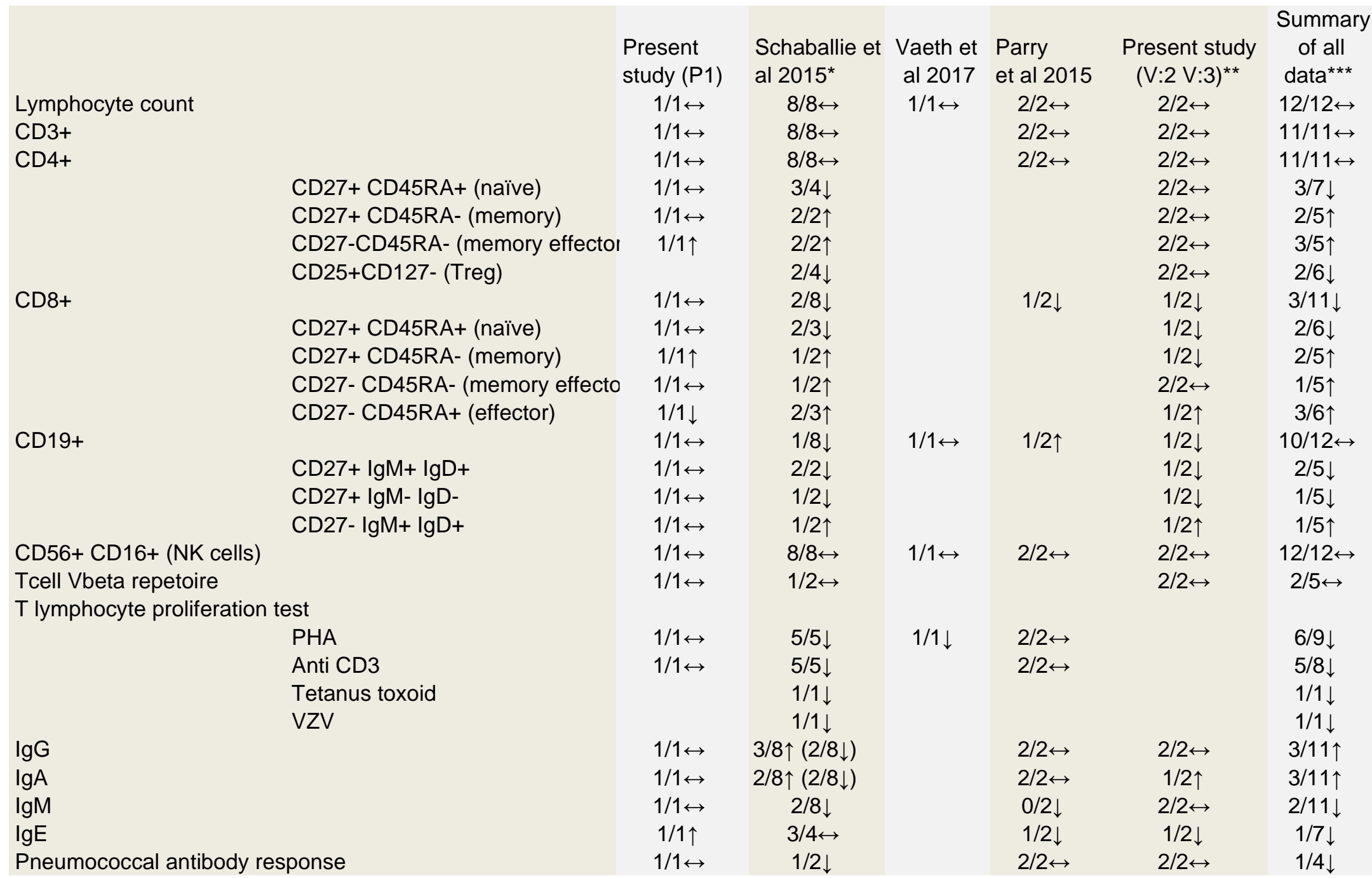

*Includes patients from Picard et al 2009, Byun et al 2010 and Fuchs et al 2012 ; ${ }^{*} \mathrm{~V}: 2$ and V:3 originaly reported by Parry et al in 2015 , follow up investigation on the same patients reported in this study $(2018)^{\star \star \star}$ No immunological findings were available for Wang et al 2014 , therefore total number of patients is $12 ; \leftrightarrow$ Normal; $\downarrow$ low; $\uparrow$ high; 
Table 2 Clinical features of STIM1 deficiency

\begin{tabular}{|c|c|c|c|c|c|c|c|c|c|c|c|c|c|c|}
\hline \multirow{2}{*}{$\begin{array}{l}\text { Study } \\
\text { Patient ID from the study }\end{array}$} & \multicolumn{3}{|c|}{ Picard et al 2009} & \multirow{2}{*}{$\begin{array}{c}\text { Byun et al } 2010 \\
\text { P4 }\end{array}$} & \multicolumn{2}{|c|}{ Fuchs et al 2012} & \multirow{2}{*}{$\begin{array}{l}\text { vaanly et al } \\
\text { ont1 } \\
\text { iV:1 }\end{array}$} & \multicolumn{2}{|c|}{ Schaballie et al 2015} & \multicolumn{2}{|c|}{ Parry ey al 2015} & \multirow{2}{*}{$\begin{array}{c}\text { Vaeth et al } 2017 \\
\text { P1 }\end{array}$} & \multirow{2}{*}{$\begin{array}{c}\text { Present study } \\
\text { P1 }\end{array}$} & \multirow[t]{2}{*}{ Summary } \\
\hline & $V-1$ & $V-4$ & $V-7$ & & P5 & P6 & & P7 & P 8 & V2 & V3 & & & \\
\hline STIM1 mutation & $\mathrm{E} 136 \mathrm{X}^{*}$ & Not known & $\mathrm{E} 136 \mathrm{X}^{*}$ & $1,538-1 G>A^{*}$ & Arg429Cys & Arg429Cys* & p.Arg426Cys* & p. $165 \mathrm{P}>\mathrm{Q}^{*}$ & p. $165 \mathrm{P}>\mathrm{Q}^{*}$ & p.L74P* & p. $L 74 \mathrm{P}^{*}$ & p.L374P* & p.Ser160fs* & \\
\hline $\begin{array}{l}\text { Predicated protein } \\
\text { effect }\end{array}$ & LOE & $\mathrm{N} / \mathrm{A}$ & LOE & LOE & LOF & LOF & LOF & LOE/LOF & LOE/LOF & LOE/LOF & LOE/LOF & LOF & LOE/LOF & \\
\hline $\begin{array}{l}\text { Age at last } \\
\text { examination }\end{array}$ & 9 & 1.5 & 6 & 2 & 6 & 1.7 & 6 & 21 & 8 & 11 & 21 & 22 & 4 & \\
\hline Immunodeficiency & 1 & 1 & 1 & 1 & 1 & 1 & 0 & 1 & 1 & 0 & 0 & 1 & 1 & $10 / 13$ \\
\hline $\begin{array}{l}\text { Immune } \\
\text { dysregulation }\end{array}$ & $\begin{array}{l}\text { AIHA, ITP, } \\
\text { LN, H/S }\end{array}$ & $\begin{array}{l}\text { AlHA, ITP, } \\
\text { LN, H/S }\end{array}$ & ITP & $\begin{array}{c}\text { AlHA, LN } \\
\text { H/S }\end{array}$ & $\begin{array}{l}\text { AlHA } \\
\text { ITP }\end{array}$ & $\begin{array}{l}\text { AIHA } \\
\text { ITP }\end{array}$ & 0 & $L N, H / S$ & 0 & 0 & $\begin{array}{c}\text { Transient } \\
\text { ITP }\end{array}$ & 0 & 0 & $8 / 13$ \\
\hline $\begin{array}{l}\text { Muscular } \\
\text { hypotonia/weaknes }\end{array}$ & 1 & 1 & 1 & 0 & 1 & 1 & 0 & 1 & 1 & 0 & 0 & 1 & 1 & $9 / 13$ \\
\hline Mydriasis & 1 & 1 & 1 & 0 & 1 & 1 & 0 & 0 & 0 & 0 & 0 & NR & 1 & $6 / 12$ \\
\hline Anhydrosis & NR & NR & NR & 0 & 1 & 1 & 0 & 1 & 0 & 1 & 1 & 1 & 1 & $6 / 10$ \\
\hline $\begin{array}{l}\text { Enamel } \\
\text { hypoplasia/defect }\end{array}$ & 1 & 0 & 0 & 0 & 1 & 1 & 0 & 1 & 1 & 1 & 1 & 1 & 1 & 9/13 \\
\hline Died & 1 & 1 & 0 & 1 & 0 & 1 & 0 & 0 & 0 & 0 & 0 & 0 & 0 & $4 / 13$ \\
\hline Alive & 0 & 0 & 1 & 0 & 1 & 0 & 1 & 1 & 1 & 1 & 1 & 1 & 1 & $9 / 13$ \\
\hline Skin involvement & 0 & 0 & 0 & $\begin{array}{c}\text { Eczematous } \\
\text { lesions } \\
\text { fingers and feet }\end{array}$ & $\begin{array}{c}\text { Generalised } \\
\text { eczema }\end{array}$ & $\begin{array}{l}\text { Severe } \\
\text { eczema }\end{array}$ & 0 & Psoriasis & $\begin{array}{r}\text { Chronic } \\
\text { dermatitis }\end{array}$ & $\begin{array}{l}\text { Mild } \\
\text { eczema }\end{array}$ & 0 & 0 & $\begin{array}{l}\text { Atypical } \\
\text { eczema } \\
\text { ichythosis, }\end{array}$ & $7 / 13$ \\
\hline Gl involvement & 0 & 0 & 0 & 0 & Colitis & 0 & 0 & Colitis & 0 & 0 & 0 & 0 & 0 & $2 / 13$ \\
\hline Nail involvement & 0 & 0 & 0 & 0 & Nail dysplasia & 0 & 1 & 0 & Brittle nails & 0 & 0 & 0 & $\begin{array}{c}\text { Nail } \\
\text { dystrophy }\end{array}$ & $3 / 13$ \\
\hline
\end{tabular}

* homozygous; ** age given in years: LOF-Loss of function; LOE-loss of expression; 1-yes; 0 no; AlHA-autoimmune hemolytic anemia; ITP-autoimmune thrombocytopenia; LN-lymphadenopathy; H/S- hepatosplenomegaly; NR-not reported 

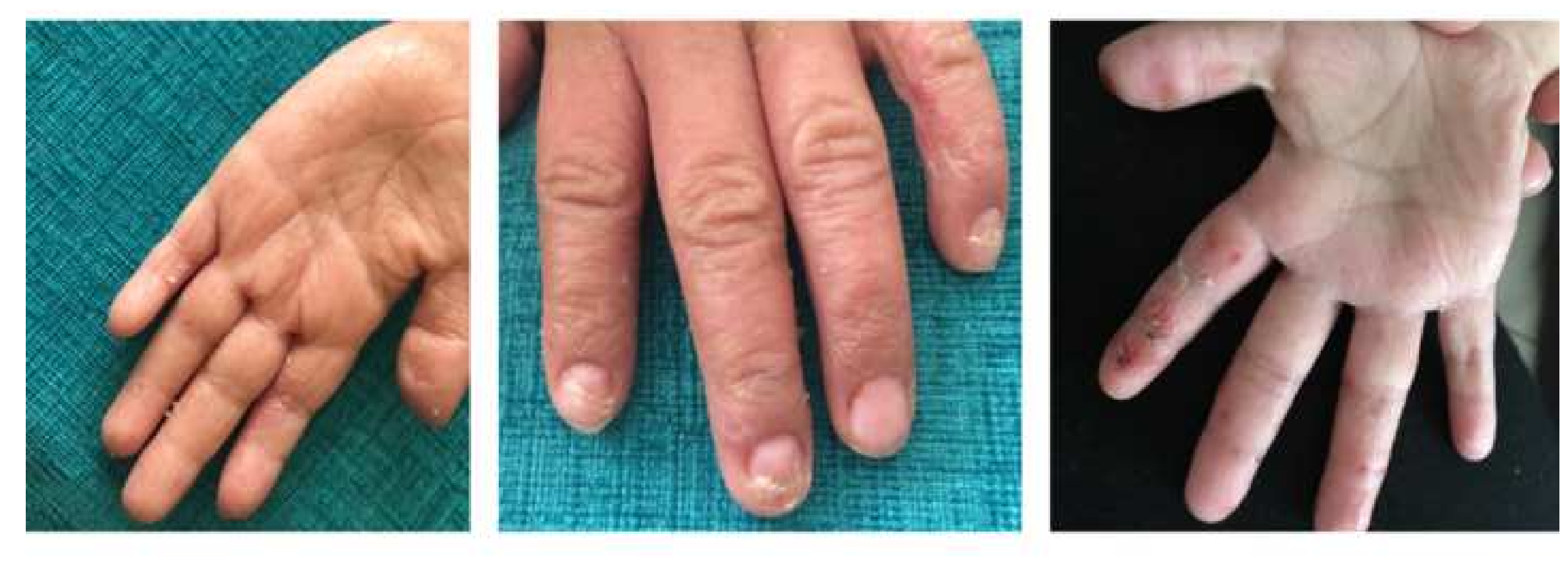


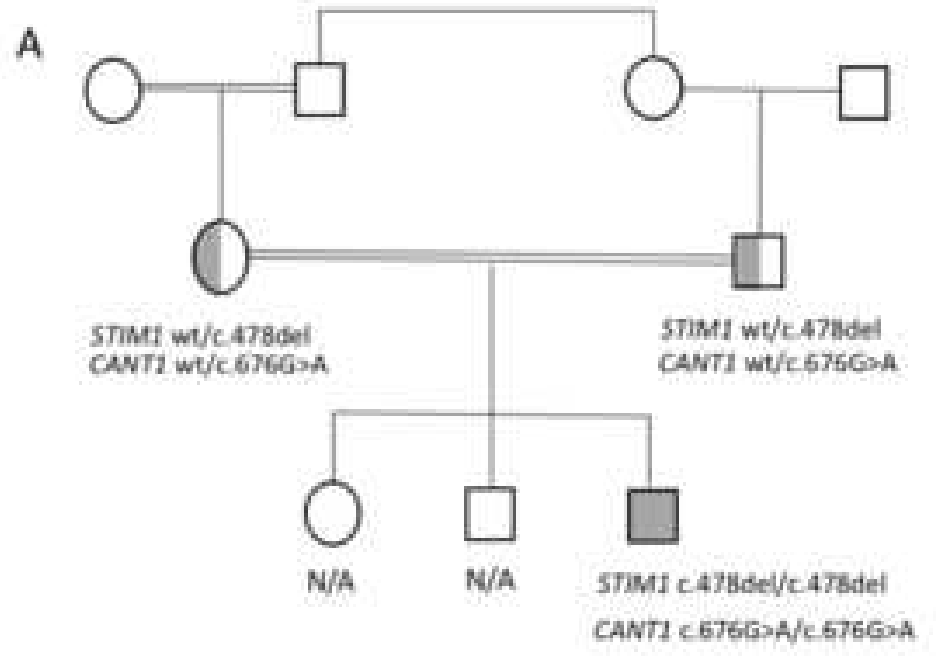

C

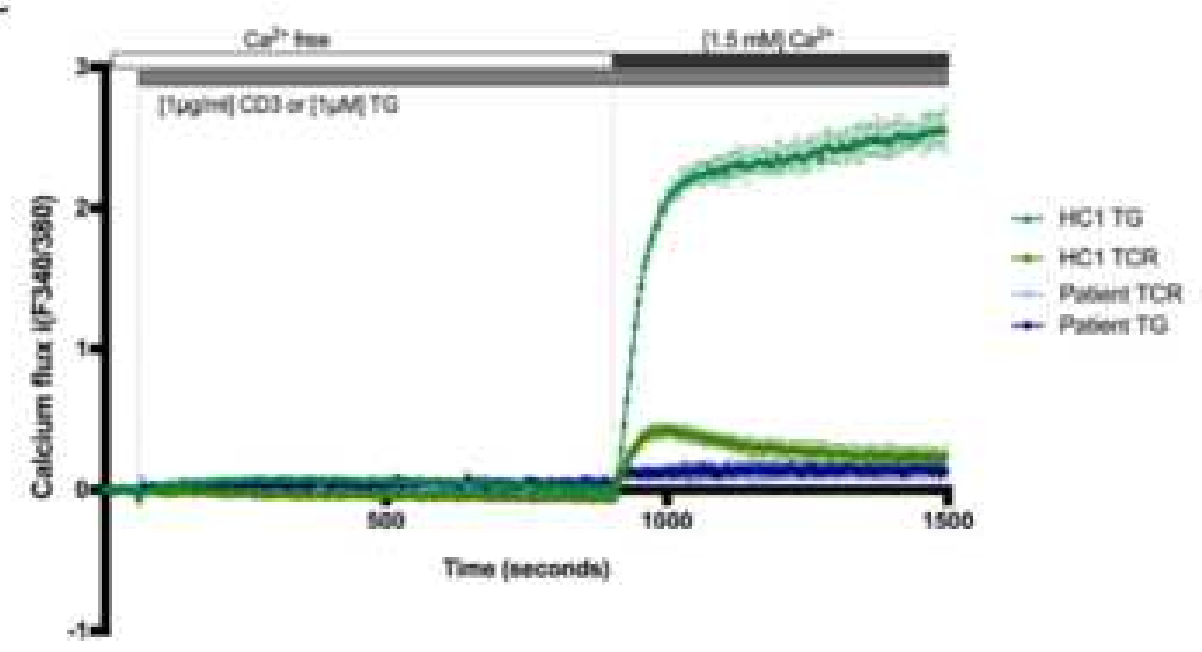

A

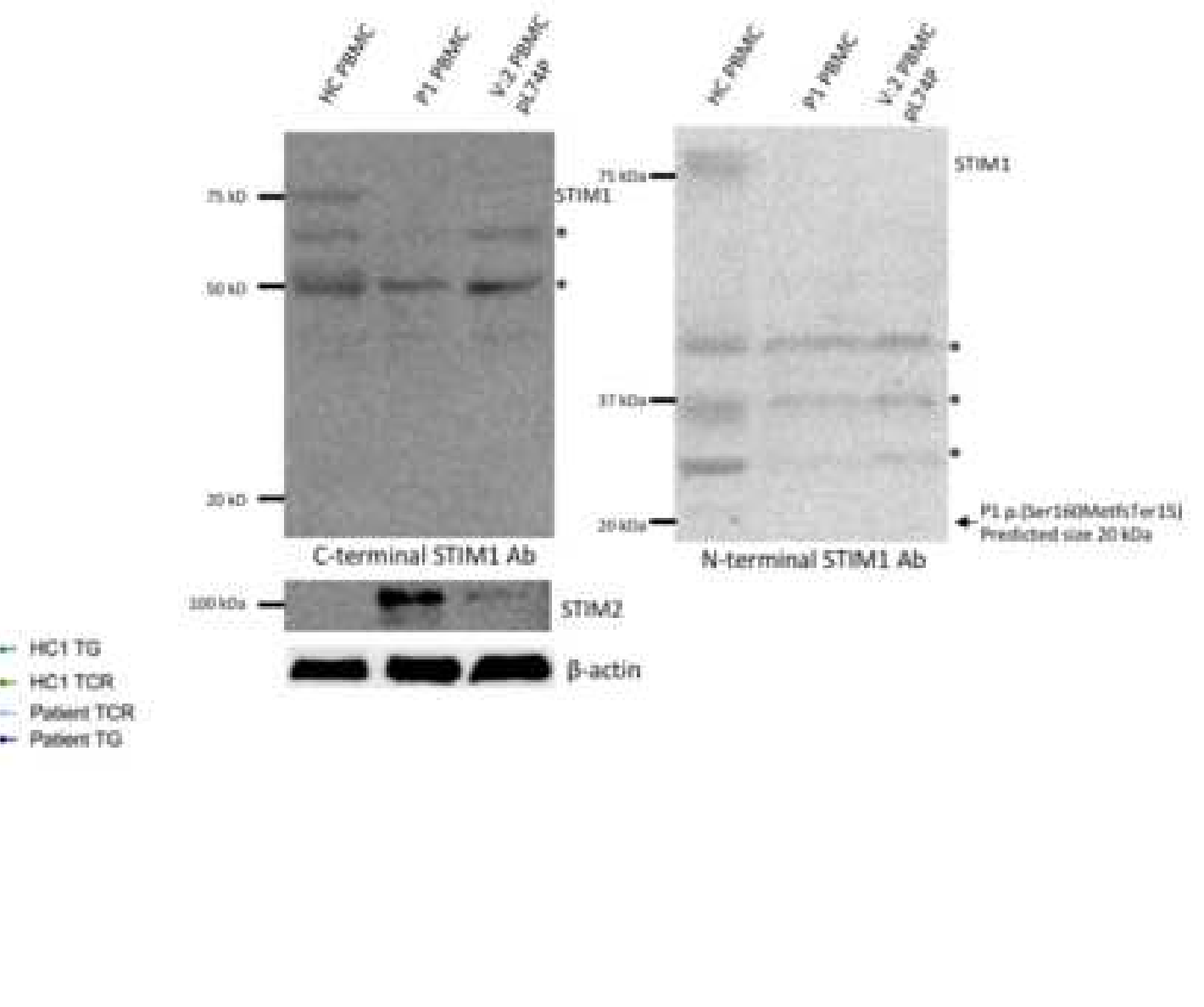



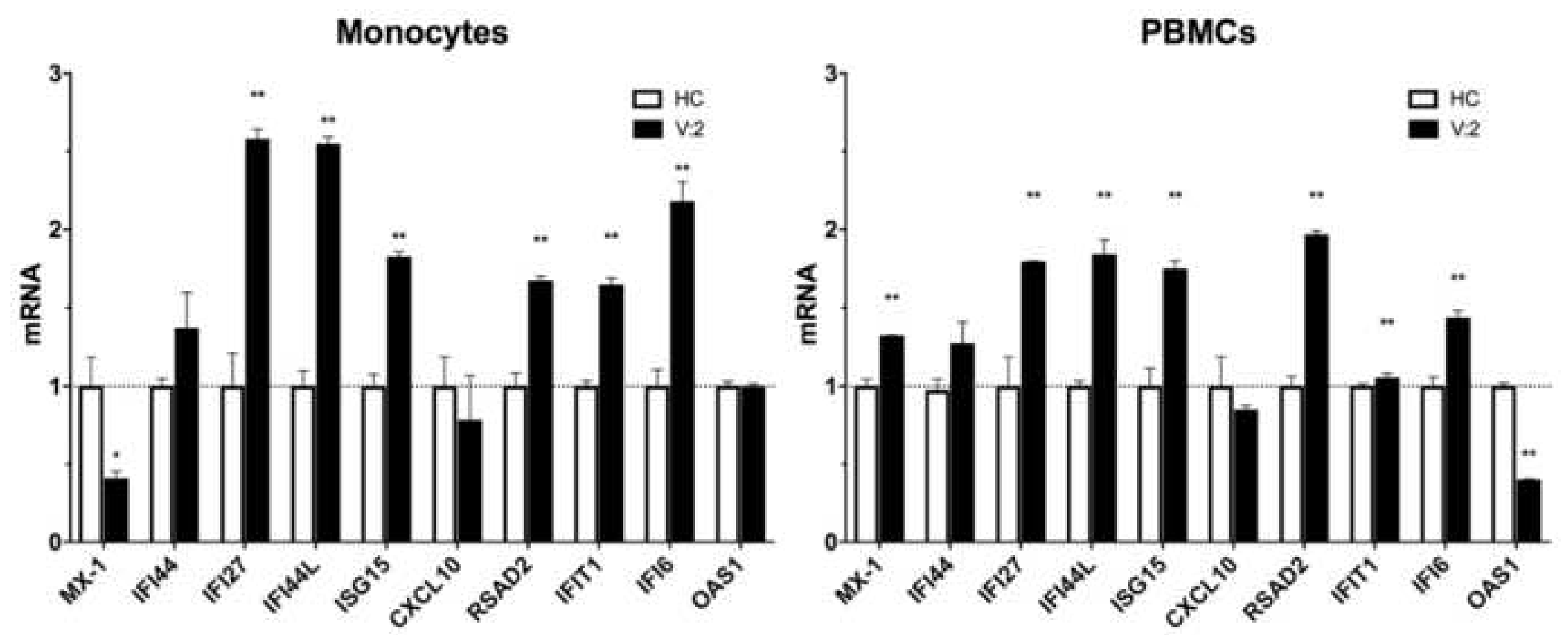